\title{
Non-scanning systems for far-infrared radiation detection from laser-induced plasmas
}

\author{
Chun $\mathrm{Li}^{1}$, Guo-Qian $\mathrm{Liao}^{1}$, and Yu-Tong $\mathrm{Li}^{1,2}$ \\ ${ }^{1}$ Beijing National Laboratory of Condensed Matter Physics, Institute of Physics, CAS, Beijing 100190, PR China \\ ${ }^{2}$ IFSA Collaborative Innovation Center, Shanghai Jiao Tong University, Shanghai 200240, PR China \\ (Received 29 January 2015; revised 16 April 2015; accepted 22 April 2015)
}

\begin{abstract}
With the development of ultraintense terawatt (TW) and petawatt (PW) laser systems, powerful terahertz (THz) radiation from laser-plasma interactions has been reported. Plasma-based THz systems, which are usually operated at extremely low repetition rates, call for single-shot diagnostics. In this paper, various state-of-the-art single-shot detection methods are introduced or designed for measurements and applications involved in high-power plasma-based THz sciences.
\end{abstract}

Keywords: laser-plasma interaction; single-shot detection; terahertz radiation

\section{General review on $\mathbf{T H z}$ detectors}

Nowadays, detection of radiation in the terahertz $(\mathrm{THz})$ frequency domain is of great interest in active research ${ }^{[1,2]}$. Generally speaking, the methods involved can be mainly categorized into two types: incoherent (direct) detection and coherent detection. In order to meet the requirements of different applications, designs for specific $\mathrm{THz}$ radiation detection systems need to take the following three factors into account: (1) whether the system has sufficiently high sensitivity to allow signals to be extracted from thermal background radiation; (2) whether the data sampling rate allows real-time measurements of ultrafast processes; and (3) whether full characterization of the $\mathrm{THz}$ radiation, including amplitude and phase information, can be provided with no or little distortion. The last requirement cannot be achieved other than by using coherent detectors/methods.

Spectral information is always considered essential in real applications. For applications that require very high spectral resolution within a known frequency region, heterodyne detection systems based on frequency mixing are preferred. At room temperature, a semiconductor-based detector, such as a planar Schottky-diode mixer, is combined with a local oscillator for frequency downconversion ${ }^{[3,4]}$. The created downshifted signal - the intermediate-frequency (IF) signal - is then filtered and amplified to obtain high sensitivity of detection, the noise equivalent power (NEP) of which

Correspondence to: Y.-T. Li, Laboratory of Optical Physics, Institute of Physics, Chinese Academy of Sciences, Beijing 100190, PR China. Email: ytli@iphy.ac.cn is typically on the order of $10^{-19} \mathrm{~W} / \sqrt{ } \mathrm{Hz}$. Cryogeniccooling techniques are used in heterodyne systems to provide even smaller NEPs of $10^{-21}-10^{-20} \mathrm{~W} / \sqrt{ } \mathrm{Hz}$. The most widely used superconducting heterodyne detector includes the superconductor-insulator-superconductor (SIS) tunnel junction mixer ${ }^{[5]}$. In principle, the detectable frequency range of a heterodyne system is determined by the operating frequency of the local oscillator, which is normally in the sub- $\mathrm{THz}$ or low- $\mathrm{THz}$ frequency region. The corresponding response time is on the level of picoseconds.

For broadband detection, direct detectors based on thermal absorption are widely used. Room-temperature thermal-type detectors include pyroelectric detectors and Golay cells. Bolometers can also be operated at room temperatures, though helium-cooling technologies are always provided to reduce background thermal noise. Compared with heterodyne devices, direct detectors have typical response times of milliseconds and suffer from a much higher NEP value (typ. $10^{-10} \mathrm{~W} / \sqrt{ } \mathrm{Hz}$ for room temperature; $10^{-12}-10^{-15} \mathrm{~W} / \sqrt{ } \mathrm{Hz}$ for helium-cooled systems). The responsivity of a thermal-type direct detector usually shows little dependence on radiation wavelength; hence bandpass filters or interferometric measurements are generally involved in providing spectral information of the measured signals. A Fourier transform infrared (FTIR) spectrometer, which is commonly used for the identification and analysis of materials with frequency structures in the $\mathrm{THz}$ domain, is operated in conjunction with a far-infrared (FIR) optical interferometer and a direct detector. Moreover, since FIR interferometry is based on the autocorrelation effects of $\mathrm{THz}$ 
(a)

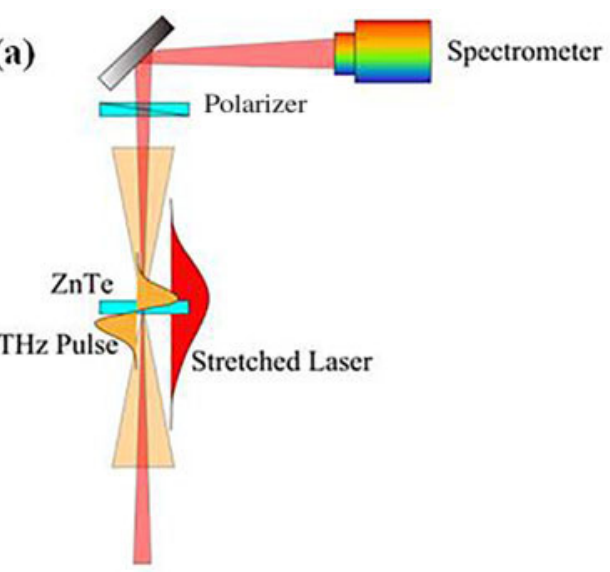

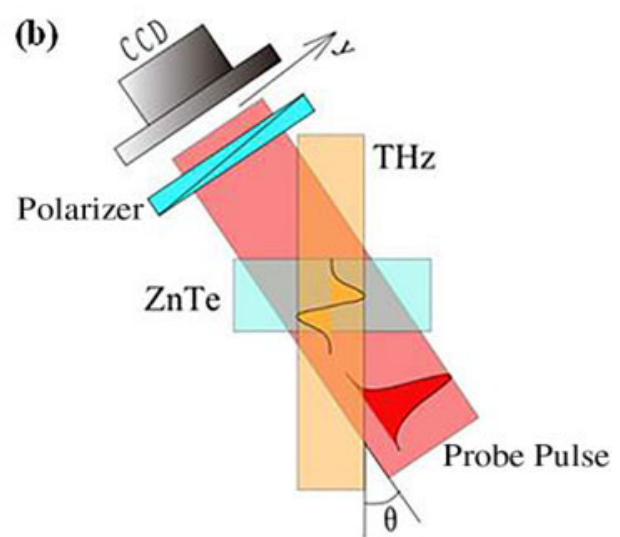

Figure 1. Schematic of (a) spectral encoding and (b) spatial encoding scheme.

pulses, it can also be used to measure the pulse duration of FIR signals ${ }^{[6]}$.

With the rapid development of ultrafast laser systems, pulsed detectors based on photoconductive or free-space electro-optic (EO) sampling are generally used for coherent detection of broadband $\mathrm{THz}$ radiation. The detection scheme excludes the majority of background noise from the time measurement window, and the signal-noise ratio (SNR) of detection surpasses $10^{4}$ using lock-in technologies ${ }^{[7]}$. Comparing photoconductive- and EO-based detection systems, the spectral response of the former is affected by the carrier lifetime as well as by antenna structures ${ }^{[8]}$, while the latter, as a pure optical technique, is considered more reliable in providing a flat frequency response over a wider frequency domain by using short probe pulses. Detectable frequencies in excess of 20 and $100 \mathrm{THz}$ have been demonstrated using photoconductive- ${ }^{[9]}$ and EO-sampling ${ }^{[10]}$ detectors, respectively. THz time-domain spectroscopy (THz-TDS), which has been developed on the basis of pulsed $\mathrm{THz}$ techniques, is now widely used to study material properties and transient processes in the FIR region.

The effective spectral response range of EO detection systems is ultimately determined by ${ }^{[11]}$ : (1) the group-velocity mismatch (GVM) between $\mathrm{THz}$ and probe pulses; and (2) the phonon absorption of EO crystals. $\mathrm{ZnTe}$ and $\mathrm{GaP}$ are two of the most commonly used sensors for EO sampling. The former shows excellent detection sensitivity around $2 \mathrm{THz}$ due to the small GVM of optical/THz pulses within the tuning range of Ti:sapphire lasers ${ }^{[12]}$, while the latter has a high fundamental transverse-optical (TO) phonon resonance frequency near $11 \mathrm{THz}^{[13]}$. Generally speaking, thinner EO crystals tend to provide a wider spectral response at the cost of detection sensitivity. The effective use of a $\langle 110\rangle$-cut $\mathrm{ZnTe}$ crystal is narrowed by about $40 \%$ (from 4 to $2.5 \mathrm{THz}$ ) as the crystal thickness is changed from 0.2 to $1 \mathrm{~mm}^{[14,15]}$. In considering the trade-off between high detection sensitivity and broadband spectral response, the type and thickness of EO crystals should be chosen carefully for specific research.

\section{Non-scanning schemes based on EO sampling}

One of the main drawbacks of conventional EO-sampling techniques is the low acquisition rate. Normally, the readout time for a single $\mathrm{THz}$ waveform would be at least seconds using serial acquisition schemes, which limits the application of this technique for real-time measurements of fast-moving objects or for real-time imaging. Moreover, how to maintain a high SNR of detection while operating the lock-in amplifier with a low time constant has always been an issue in fast scanning ${ }^{[7]}$.

To increase the data acquisition rate, conventional singlechannel delay-scanning detection should be replaced by parallel data collection and multi-channel detector arrays. Much effort has been made in developing 'single-shot' (nonscanning) EO detection systems since 1998, and in general the techniques are progressing in two directions: spectral encoding $^{[16]}$ and spatial encoding ${ }^{[17]}$; see Figures 1(a) and 1(b), respectively. The main idea is to record the entire temporal profile of a $\mathrm{THz}$ pulse onto the spectral or spatial profile of a probe pulse for parallel data acquisition by a spectrometer or a charge-coupled device (CCD) camera. However, both schemes have obvious shortcomings. The spectral encoding method, which relies on measurement of the spectral modulation of a chirped probe pulse, has a fundamental temporal resolution limit related to the chirped pulse duration ${ }^{[18]}$. The spatial encoding method, which is based on the non-collinear geometry of a collimated short probe pulse along with a collimated $\mathrm{THz}$ pulse, retains an excellent time resolution but suffers from a much weaker sensitivity of detection. In addition, the spatial encoding method relies only on the energy/pointing stability of the laser system, while the spectral encoding scheme is also sensitive to spectral instability of the laser.

To achieve both high sensitivity and temporal resolution, a third 'single-shot' EO-cased technique has been introduced as a cross-correlation technique by second-harmonic generation $(\mathrm{SHG})^{[19]}$, as depicted in Figure 2, which can 


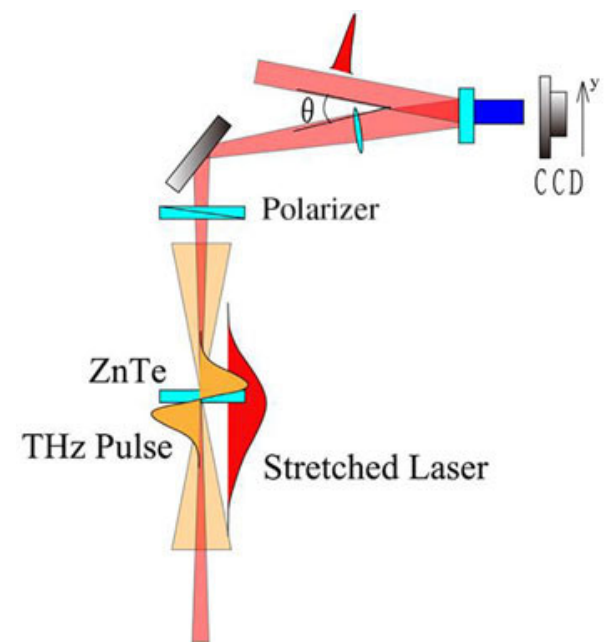

Figure 2. Schematic of 'single-shot' cross-correlation scheme.

be considered as a combination of the spectral and spatial encoding techniques. However, since a second probe pulse is necessary, the complexity of the setup limits the applicability of this technique in real applications. In order to improve the temporal resolution while retaining the simplicity of measurements included in the spectral encoding technique, a different retrieval algorithm has been applied to the experimental setup depicted in Figure 1(a). The corresponding technique is referred to as 'single-shot' in-line spectral interferometry ${ }^{[20,21]}$, by which the THz field will be derived from the measured optical spectral modulation through a matrix inversion. However, the algorithm cannot be applied in experiments which involve substantial noise.

Based on the schemes (including algorithms) shown in Figures 1 and 2, proof-of-principle experiments have been conducted on quasi-continuous-wave lasers ${ }^{[16,17,19,20]}$ (operated at $\mathrm{kHz}$ or $\mathrm{MHz}$ ). Since at least two laser shots are needed to identify the modulation caused by the $\mathrm{THz}$ field, the accuracy of signal recovery depends strongly on the stability of the laser system. Sequential acquisition of the signal and reference pulses will prevent the use of such techniques in high-energy laser systems, which usually operate at low repetition rates and are accordingly less stable.

\section{True single-shot $\mathbf{T H z}$ diagnostics in intense laser- plasma interactions}

The development of $\mathrm{THz}$ sciences is in great need of highpower $\mathrm{THz}$ sources. With the rapid development of ultrashort laser systems, laser-plasma interactions as a new field of research have received considerable attention for strong $\mathrm{THz}$ radiation generation ${ }^{[6,22-26]}$. However, with increasing laser energies, the repetition rates and stability of laser systems are both decreased. The sensitivity of laser-plasma interactions to small laser fluctuations has motivated the development of true single-shot THz-diagnostic techniques.

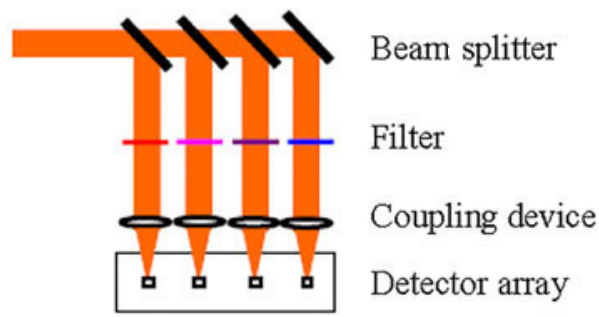

Figure 3. Schematic of a multi-channel THz-diagnostic system based on a direct detector array.

\subsection{Incoherent detection}

Direct measurements based on thermal absorption are still by far the most convenient and widely used methods for the detection of broadband plasma-based $\mathrm{THz}$ sources. In order to obtain spectral information in one laser shot, multichannel detection with bandpass or low-pass filters is preferred, as depicted in Figure 3. The detector array usually consists of a number of identical pyroelectric elements; or in the case of sufficient radiation flux, a pyroelectric camera can be considered as a receiver. Selectable beam splitters include silicon wafers and pellicles; coupling devices can be from a wide range of $\mathrm{THz}$ lenses and waveguides. By deliberate calibration of the split ratios, coupling coefficients, filter transmission curves and detector response curves, the essential spectral information of $\mathrm{THz}$ pulses can be obtained from one laser shot. This multi-channel THz-diagnostic system provides a convenient way to avoid shot-to-shot laser fluctuations, and offers the flexibility of selecting the frequency range of interest simply by changing the $\mathrm{THz}$ filters.

\subsection{Coherent detection}

$\mathrm{THz}$ pulses generated from ultrafast laser-plasma interactions are often characterized by pump and probe measurements. Traditional pump-probe schemes such as EO sampling, which relies on lock-in techniques, are very sensitive to laser noise (which can be quite substantial at low repetition rates) and low-frequency disturbance. For intense laserplasma interactions, the low repetition rate and relatively large laser fluctuations have motivated the development of true single-shot EO-based measurements. In principle, the non-scanning schemes introduced in Section 2 can be used in a single-shot measurement if a reference beam be provided along with the probe beam in one laser shot for direct comparison ${ }^{[25]}$. In this section, two further singleshot techniques via temporal-spectral or temporal-spatial conversion will be introduced.

3.2.1. Single-shot interferometric scheme using frequencydomain holography

Spectral interferometry, which is also called Fourier-domain interferometry (FDI), is a well-known linear technique for 


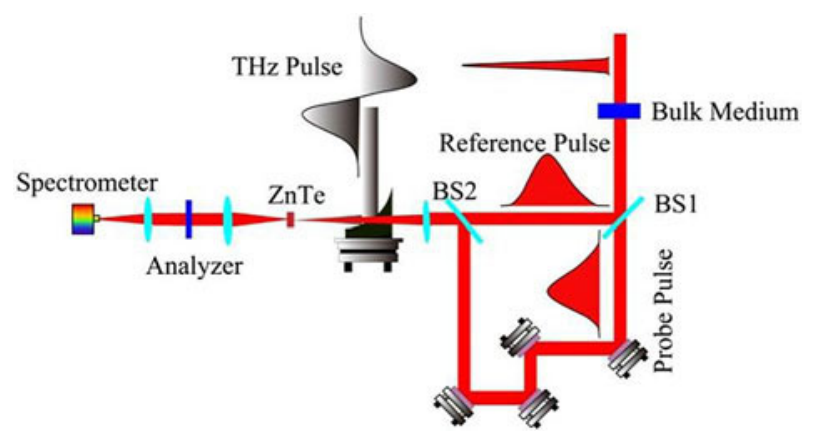

Figure 4. Spectral interferometry scheme using twin-chirped-pulse FDH (quoted from Ref. [28]).

phase and amplitude retrieval from an unknown field in the femtosecond domain ${ }^{[27]}$. Normally, multiple shots are required to obtain the complete temporal field, but the acquisition rate can be greatly increased by using linearly chirped probe pulses. The in-line interferometric scheme is considered unsuitable for true single-shot $\mathrm{THz}$ field diagnostics, since a reference shot has to be recorded as the background before performing the signal retrieval ${ }^{[20]}$. We proposed a modified scheme using single-shot frequencydomain holography $(\mathrm{FDH})^{[28]}$, as depicted in Figure 4. The original femtosecond optical pulse is frequency chirped and time stretched to several picoseconds before splitting by a $50 / 50$ beam splitter (BS1). A fixed time delay $\tau$ is introduced to overlap the probe pulse within the duration of the $\mathrm{THz}$ pulse in the ZnTe crystal. The delayed and modulated probe pulse then interferes with the reference pulse in an imaging spectrometer, and the interferometric fringes are recorded on a CCD camera. The interference pattern, with a period proportional to $2 \pi / \tau$, which contains information on the phase and amplitude modulation caused by the $\mathrm{THz}$ field, will then be analyzed to retrieve the $\mathrm{THz}$ waveform in a single shot. The temporal resolution of this technique relies on the retrieval algorithm. If the signal recovery is based on the linear chirped time-frequency relation, a temporal limit identical to that of the spectral encoding method will be given by the uncertainty principle ${ }^{[18]}$. The implementation of such a technique requires use of a highresolution spectrometer.

3.2.2. Single-shot THz-pulse characterization by dual echelon optics

In the 2000s, dual echelon optics was first employed to single-shot $\mathrm{THz}$ diagnostics ${ }^{[29]}$. The main idea is to divide the transverse profile of a probe beam by stair-step (echelon) structures into multiple delayed beamlets, which are then focused onto the same spot on an EO crystal to monitor the phase modulation caused by a copropagating $\mathrm{THz}$ pulse. The portions of probe beamlets with $\mathrm{THz}$ temporal information encoded on them are sequentially imaged onto different positions of a CCD camera for single-shot image processing.

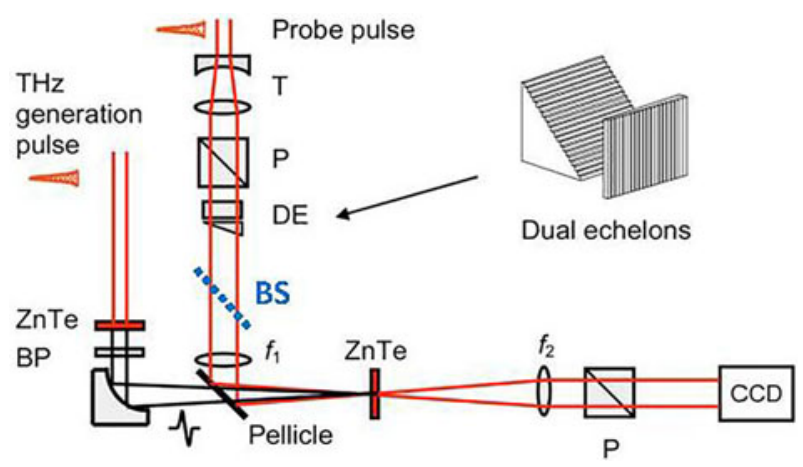

Figure 5. Schematic of single-shot $\mathrm{THz}$ measurement using dual echelon optics (quoted from Ref. [29]).

To maximize the number of sampling points within one laser shot, two orthogonally oriented echelons are used (rather than one) to fully subdivide the transverse probe beam profile, which has been pre-magnified in diameter. The so-called echelon optics technique, as depicted in Figure 5, which uses a collinear geometry with focused copropagating $\mathrm{THz}$ and probe beams, maintains a high detection sensitivity while providing high time resolution and a large time window. For a true single-shot measurement, a beam splitter (blue dot line in Figure 5) should be applied in the optical path to provide an additional reference pulse which bypasses the ZnTe crystal. The reference image is used for direct comparison with the signal image within one laser shot.

\subsection{Infrared streak camera}

Most electronic devices cannot be used for the direct measurement of fast transient processes in the picosecond region, with the exception of streak cameras. The spectral response of a streak camera is determined by the photocathode material, most of which are sensitive only to electromagnetic radiation of wavelengths shorter than $1.5 \mu \mathrm{m}$. By using gasphase Rydberg atoms as the cathode, the measurable wavelength can be extended to the FIR region ${ }^{[30]}$. Alternatively, by placing an EO crystal as a converter before an optical streak camera, $\mathrm{THz}$ pulses can be retrieved indirectly by measuring copropagating long probe pulses ${ }^{[31]}$. Comparing an atomic streak camera and an EO-based infrared streak camera, the former can provide information only on the time envelope of $\mathrm{THz}$ waves, while the latter is in principle a coherent measurement (but requires an additional reference shot for $\mathrm{THz}$ field diagnosis). The temporal resolution of infrared streak camera systems is not comparable to that which can be achieved by single-shot EO-based optical systems.

\section{Summary and outlook}

In this paper, we present an overview on the state-of-theart techniques for single-shot $\mathrm{THz}$ diagnostics involved in 
intense laser-plasma interactions. For incoherent detection, direct detectors can be used with $\mathrm{THz}$ filters to provide general spectral information. Atomic infrared streak cameras, which operate in the infrared regime (typ. from 1 to $100 \mu \mathrm{m})$, are able to measure the intensity profile of $\mathrm{THz}$ pulses directly, with a time resolution of about one picosecond. For coherent detection, a THz field can be retrieved via single-shot EO-sampling techniques, either by time-to-space or time-to-spectrum conversion. Associated with specific applications, THz-diagnostic systems should be carefully designed according to the experimental and laser conditions. Practically speaking, high detection sensitivity is always the first concern, followed by high time resolution. Efforts toward higher-power $\mathrm{THz}$ sources and an improved understanding of $\mathrm{THz}$ radiation under extreme conditions, would promise continuing advances in single-shot $\mathrm{THz}$ technologies for intense laser-plasma interactions.

\section{Acknowledgements}

This work is supported by the National Basic Research Program of China (grant no. 2013CBA01501), the National Nature Science Foundation of China (grant nos. 11135012 and 11375262), and the Key Research Program of the Chinese Academy of Science (grant no. KGZD-EW-T05).

\section{References}

1. B. Ferguson and X.-C. Zhang, Nat. Mater. 1, 26 (2002).

2. P. H. Siegel, IEEE Trans. Microwave Theory Technol. 50, 910 (2002).

3. S. Tzortzakis, G. Méchain, G. Patalano, Y.-B. André, B. Prade, M. Franco, A. Mysyrowicz, J.-M. Munier, M. Gheudin, G. Beaudin, and P. Encrenaz, Opt. Lett. 27, 1944 (2002).

4. M. C. Gaidis, H. M. Pickett, C. D. Smith, R. P. Smith, S. C. Martin, and P. H. Siegel, IEEE Trans. Microwave Theory Technol. 48, 733 (2000).

5. G. J. Dolan, T. G. Phillips, and D. P. Woody, Appl. Phys. Lett. 34, 347 (1979).

6. H. Hamster, A. Sullivan, S. Gordon, W. White, and R. W. Falcone, Phys. Rev. Lett. 71, 2725 (1993).

7. Y. Cai, I. Brener, J. Lopata, J. Wynn, L. Pfeiffer, J. B. Stark, Q. Wu, X. C. Zhang, and J. F. Federici, Appl. Phys. Lett. 73, 444 (1998).

8. S.-G. Park, M. R. Melloch, and A. M. Weiner, Appl. Phys. Lett. 73, 3184 (1998).
9. S. Kono, M. Tani, P. Gu, and K. Sakai, Appl. Phys. Lett. 77, 4104 (2001).

10. C. Kübler, R. Huber, and A. Leitenstorfer, Semicond. Sci. Technol. 20, S128 (2005).

11. G. Gallot and D. Grischkowsky, J. Opt. Soc. Am. B 16, 1204 (1999).

12. Q. Wu and X.-C. Zhang, Appl. Phys. Lett. 68, 1604 (1996).

13. Q. Wu and X.-C. Zhang, Appl. Phys. Lett. 70, 1784 (1997).

14. J. van Tilborg, C. B. Schroeder, C. V. Filip, Cs. Tóth, C. G. R. Geddes, G. Fubiani, R. Huber, R. A. Kaindl, E. Esarey, and W. P. Leemans, Phys. Rev. Lett. 96, 014801 (2006).

15. A. Nahata, A. S. Weling, and T. F. Heinz, Appl. Phys. Lett. 69, 2321 (1996)

16. Z. Jiang and X.-C. Zhang, Appl. Phys. Lett. 72, 1945 (1998).

17. J. Shan, A. S. Weling, E. Knoesel, L. Bartels, M. Bonn, A. Nahata, G. A. Reider, and T. F. Heinz, Opt. Lett. 25, 426 (2000).

18. F. G. Sun, Z. Jiang, and X.-C. Zhang, Appl. Phys. Lett. 73, 2233 (1998)

19. S. P. Jamison, J. Shen, A. M. MacLeod, W. A. Gillespie, and D. A. Jarpszynski, Opt. Lett. 28, 1710 (2003).

20. K. Y. Kim, B. Yellampalle, G. Rodriguez, R. D. Averitt, A. J. Taylor, and J. H. Glownia, Appl. Phys. Lett. 88, 041123 (2006).

21. B. Yellampalle, K. Y. Kim, G. Rodriguez, J. H. Glownia, and A. J. Taylor, Appl. Phys. Lett. 87, 211109 (2005).

22. W. P. Leemans, C. G. R. Geddes, J. Faure, Cs. Tóth, J. van Tilborg, C. B. Schroeder, E. Esarey, G. Fubiani, D. Auerbach, B. Marcelis, M. A. Carnahan, R. A. Kaindl, J. Byrd, and M. C. Martin, Phys. Rev. Lett. 91, 074802 (2003).

23. Z.-M. Sheng, K. Mima, J. Zhang, and H. Sanuki, Phys. Rev. Lett. 94, 095003 (2005).

24. K. Y. Kim, A. J. Taylor, J. H. Glownia, and G. Rodriguez, Nat. Photonics 2, 605 (2008).

25. Y. T. Li, C. Li, M. L. Zhou, W. M. Wang, F. Du, W. J. Ding, X. X. Lin, F. Liu, Z. M. Sheng, X. Y. Peng, L. M. Chen, J. L. Ma, X. Lu, Dong Z. H. Wang, Z. Y. Wei, and J. Zhang, Appl. Phys. Lett. 100, 254101 (2012).

26. A. Gopal, S. Herzer, A. Schmidt, P. Singh, A. Reinhard, W. Ziegler, D. Brömmel, A. Karmakar, P. Gibbon, U. Dillner, T. May, H.-G. Meyer, and G. G. Paulus, Phys. Rev. Lett. 111, 074802 (2013).

27. J.-P. Geindre, P. Audebert, S. Rebibo, and J.-C. Gauthier, Opt. Lett. 26, 1612 (2001).

28. M. L. Zhou, F. Liu, C. Li, F. Du, Y.-T. Li, W.-M. Wang, Z.-M. Sheng, L.-M. Chen, J.-L. Ma, X. Lu, Q.-L. Dong, and J. Zhang, Chinese Phys. Lett. 29, 015202 (2012).

29. K. Y. Kim, B. Yellampalle, A. J. Taylor, G. Rodriguez, and J. H. Glownia, Opt. Lett. 32, 1968 (2007).

30. M. Drabbels and L. D. Noordam, Appl. Phys. Lett. 74, 1797 (1999).

31. Z. Jiang, F. G. Sun, and X.-C. Zhang, Opt. Lett. 24, 1245 (1999). 